\title{
An Integrated Model-based Approach for FMECA Development for Smart Manufacturing Applications
}

\author{
Sudipto Ghoshal ${ }^{1}$, Somnath Deb ${ }^{2}$, Deepak Haste ${ }^{3}$, Andrew Hess ${ }^{4}$, Feraidoon Zahiri ${ }^{5}$ and Gregory Sutton ${ }^{6}$ \\ ${ }^{1,2,3}$ Qualtech Systems, Inc., Rocky Hill, CT 06067, USA \\ sudipto@teamqsi.com \\ deb@teamqsi.com \\ deepak@teamqsi.com \\ ${ }^{4}$ Hess PHM Group, Easton, VA, USA \\ andrew.hess@comcast.net \\ 5,6402d Commodities Maintenance Group, Warner-Robins Air Logistics Complex, Robins AFB, GA, USA \\ feraidoon.zahiri@us.af.mil \\ gregory.sutton@us.af.mil
}

\begin{abstract}
Qualtech Systems, Inc. (QSI)'s integrated tool set, consisting of TEAMS-Designer ${ }^{\circledR}$ and TEAMS-RDS ${ }^{\circledR}$ provides a comprehensive model-based systems engineering approach that can be deployed for fault management throughout the equipment life-cycle - from its design for fault management to condition-based maintenance of the equipment. The TEAMS ${ }^{\circledR}$ failure-cause effect dependency model is a digital twin representation of the equipment in its failure-space and allows for various types of analyses such as testability, serviceability, failure propagation and others that facilitate fault management design of the equipment. The same model is deployed through TEAMS-RDS ${ }^{\circledast}$ for condition monitoring, prognostics, real-time health assessment, failure impact analysis, guided troubleshooting and others that facilitate condition-based maintenance as well as ensure efficient and rapid maintenance actions. In this paper, we present an overview of QSI's integrated toolset, with a focus on a systematic model-based approach towards an automated development of Failure Effects and Criticality Analysis (FMECA) and other relevant analyses for the equipment, for an improved understanding of failure effects and their causality at the system-level. The eventual objective here is improved equipment design as well as designing improved failure detection, failure isolation and failure mitigation. The paper will also discuss examples of such real-world
\end{abstract}

Sudipto Ghoshal et al. This is an open-access article distributed under the terms of the Creative Commons Attribution 3.0 United States License, which permits unrestricted use, distribution, and reproduction in any medium, provided the original author and source are credited. applications for smart manufacturing in major depot maintenance facilities in the US. A subsequent paper will focus on the development and integration of process-level and equipment-level FMECAs for Smart Manufacturing applications.

\section{INTRODUCTION}

As technology advances, there is a significant increase in the complexity, sophistication and automation of manufacturing processes and correspondingly, of the systems and equipment that are key constituent elements of the manufacturing processes. A modern automated manufacturing process consists of sets of different complex equipment working sequentially on the products being manufactured as they make their way through the processing line to their desired form, fit and finish. Each of these manufacturing processing lines with intricate dependencies between the process and the equipment results in a challenging environment for timely failure detection, accurate failure identification and corresponding mitigation.

As an example, consider a modern automated metal finishing facility for aircraft parts manufacturing or overhaul. The controllers for such manufacturing processes execute a large set of well-orchestrated sequence of processes such as cleaning, etching, rinsing, metal stripping, deoxidizing or $\mathrm{pH}$ neutralizing, coating, plating and others. Each of these processes executed correctly in sequence leads to the finished metal product that can pass various acceptance tests including visual inspections, corrosion resistance checks, adhesion tests etc. Any failure of these tests can lead to the rejection of not 
just that specific part but can also lead to the condemnation of the entire batch making such failures extremely expensive. The above failures can occur due to a whole host of factors that may include environmental factors such as incorrect temperature control, humidity control and process-related factors such as inadequate processing times, incorrect solution composition and contamination, as well as equipment-level failures which may have direct causal relationship with the aforementioned factors.

A thorough understanding of these failures and their effects on the finished product is essential in order to set up appropriate monitoring and preventive management of those failure effects. Thus, the step of understanding and documenting process-level failure modes and their effects is the first key step in ensuring that a failure management system can be put in place that ensures a finished product satisfies all requirements and passes all acceptance tests for it to be installed in the aircraft. Failures that are not understood will likely not be adequately monitored, detected, isolated and corrected in a timely manner. This can lead to significant loss of material, time and resources for the facility and can even halt the production process. Thus, the first and foremost need that is essential for efficient and reliable operations at any modern manufacturing facility is a comprehensive understanding of the different potential failures in all of the processing stages that can lead to the product failing to meet its form, fit and function related specifications.

This paper (part of a series of two) describes QSI's causeeffect multi-function model-based systematic approach towards automated development of documents such as the FMECA, Dependency Analysis and Fault Trees for the identification of equipment-level failures, their propagation through the manufacturing system and the manifestation of those failures as undesirable effects during equipment operation. The paper also provides a brief description the TEAMS $^{\circledR}$ software products from QSI that utilize that modeling methodology. The same cause-effect dependency models are used by QSI's TEAMS ${ }^{\circledR}$ software products for systematic analyses for overall fault management design as well as operational-level process and equipment health monitoring and guided troubleshooting in the event of failures. A subsequent paper will focus on process-level FMECA development and its integration with equipmentlevel FMECA as described in this paper for the development of an automated overall system-level FMECA that can be applied towards large and complex manufacturing processes. QSI is currently adapting and leveraging its TEAMS $^{\circledR}$ software tools for integrated process-level and equipmentlevel FMECAs for a major smart manufacturing facility in the US and the paper will conclude with the modeling methodology adapted for the automatic generation and

\footnotetext{
${ }^{1}$ This work was supported in part by Air Force (AFWERX) Phase II SBIR (FA875119CA054).
}

update of FMECA for the process and equipment at that facility ${ }^{1}$. The FMECA will lead to the subsequent adaptation of the TEAMS ${ }^{\circledast}$ model for process and equipment health assessment and facilitate guided troubleshooting, as necessary, for failure root-cause isolation and mitigation/recovery. It is anticipated that the same modeling methodology and software integration and deployment strategy, if proven to be successful at this facility, can be readily adapted for other similar smart manufacturing centers.

\section{QSI'S INTEGRATED TEAMS ${ }^{\circledR}$ TOOLSET}

Increasing system and process complexity and automation presents formidable system health management related challenges to manufacturers and end users of complex systems, such as aircraft, helicopters, spacecraft as well as smart manufacturing centers. Qualtech Systems Inc.'s testability engineering tool, TEAMS ${ }^{\circledR}$, employed dependency modeling, albeit in a hierarchical directed graph format, to model such complex systems. The TEAMS ${ }^{\circledR}$ modeling approach allows the modeler to capture system information more naturally in a "colored" di-graph format that retains close relationship to the structure or information flow in a system. QSI has also developed additional software tools that expand the TEAMS $^{\circledR}$ family for comprehensive fault management design and analysis and also for operational usage such as runtime health assessment and efficient field maintenance service. Our current research is focused on developing an integrated package with comprehensive diagnostics and prognostics capabilities that addresses Design for Testability (DFT) and Serviceability, FMECA, Fault Tree Analysis as well as Condition-Based Maintenance and Guided Troubleshooting. The use of the same model for all of the above analyses and maintenance-related services eliminates redundant modeling and ensures that the detection and isolation measures predicted in the design phase are realized in the field.

\section{TEAMS ${ }^{\circledR}$ MODELING METHODOLOGY}

Minimizing the life-cycle cost of a complex system requires a well-coordinated effort involving people of different expertise. In effect, the model is the means by which people document and convey their understanding of the system, as it relates to their fields of expertise. For example, to the design engineer, the model could be a block diagram with transfer functions, whereas to a maintenance engineer, it is the schematic of replaceable components that make up the system. The objective is to develop a modeling methodology that is simple and intuitive enough so that people of various 
disciplines can understand and relate to it, yet powerful enough to be used during the entire life-cycle of a system.

The foundation of TEAMS ${ }^{\circledR}$ modeling methodology is based on the following concepts:

First, for diagnostic purposes, we only need to model how a fault (or cause) propagates to the various monitoring points. The objective is not design verification: we assume that the system normally works to specification. The failure of one or more components (causes) results in system malfunctions (effects) that are observable at various points (test points) in the system. For FMECA, the goal is to trace the effects of a failure and assess its impact on system performance. For DFT, the goal is to ensure that the system is sufficiently observable (and controllable) so that the cause of a malfunction can be easily identified. In field maintenance, the goal is to identify the cause of a malfunction in minimum time/cost. In all these cases, it is sufficient to model the system in its failure space. Thus, the system can be modeled in terms of first-order cause-effect dependencies, i.e., how a faulty node affects its immediate neighbors. Higher-order dependencies can be inferred from first-order dependencies.

Second, the failure space is not binary (i.e., simple pass/fail), as is assumed in structural and traditional dependency models. The function space is multidimensional. Consequently, the failure space, which is the complement of function space, is also multidimensional. For example, the function of a sine wave generator is to generate a sine wave of specified amplitude, phase and frequency. It is said to have failed if the output sine wave does not have any of the desired amplitude, phase or frequency.

Third, since the failure state can be arbitrary, it is unnecessary to model the exact quantitative relationships. In order to illustrate this assertion, consider a cascade of three amplifiers, having gains of 2, 3, and 4, with an overall gain of 24. If, due to a fault, the new gain is 12, the first stage, with a design gain of 2 , should not necessarily be implicated; the gain of any of the stages may have been reduced by half due to a failure. Thus, when the same attribute is modified by multiple components, quantitative relationships convey little, if any, information. If the gain is off, all of the amplifiers should be included as likely suspects and further testing that can isolate the effects of the individual amplifier is necessary for identifying the failed amplifier(s). So, it is only necessary to identify the important functional attributes (or the dimensions of the function space) and associate them with the appropriate components and tests.

Fourth, there can be two distinct types of failures: functional failures and general failures. Consider a lossless (passive) filter consisting of an inductor and a capacitor. If a fault in the inductor or capacitor causes a deviation in the center frequency, it is considered a functional failure, i.e., a fault that affects the function it was supposed to perform. On the other hand, if the fault is a short-circuit that causes the output power to be zero, this is a general failure, that is, a catastrophic failure affecting attributes beyond its normal functioning by interrupting the flow of information through it. Thus, a failure in a module can either affect the attributes it was supposed to (functionally) modify, or all the attributes flowing through it. This affects how the overall cause-effect dependencies are derived from the structure and function information.

Finally, the functional failures can additionally be construed as local, global or transformed. While, by default, functional failures are global, a local functional failure has a limited scope in terms of its propagation through the system and is constrained to only affect the component or its immediate component neighbors within a higher-level module and does not propagate beyond. This can be despite the presence of physical or structural connectivity that otherwise allow propagation. Thus, a locally scoped functional failure does not affect any modules/components beyond the hierarchy within which it is contained. In other cases, the functional failure may propagate but after being transformed by a downstream component into a different functional failure. A simple example is electric power being converted to torque by the motor. Hence, a failure in the Power Supply module may lead to inadequate torque which may impact downstream components which rely on adequate torque availability.

\subsection{Basic constructs in TEAMS ${ }^{\circledR}$ models}

TEAMS ${ }^{\circledR}$ modeling is a hierarchical modeling methodology, where the propagation paths of the effects of a failure are captured in terms of a directed graph. The graph has five basic types of nodes with their own sets of function and failure-space properties:

The Module corresponds to a piece of hardware with a certain set of functions (captured in terms of signals). Modules themselves can be described in terms of another graph consisting of (sub)modules and other nodes - allowing for hierarchical modeling. A module at the lowest level is called a failure mode which capture the modality of the failure of the component. The failure modality or failure mode leads to impacting the associated component function(s) in the system which propagate and affect other modules and manifests at various locations in the system. Diagnosis is the process of identifying the root-cause failure source(s) from analyzing all the failure manifestations - both their presence and absence, throughout the system.

The Test Point corresponds to locations (physical or logical) where measurements can be made that can indicate presence or absence of failure(s) in the system. A test point can have multiple tests - i.e., at a single physical location (or probe point) where one or more measurements may be made. Such tests can be classified as symptoms, diagnostic tests (manual or automatic), degradation tests for prognostics, or can be associated with levels or labels to model different echelons of maintenance. Thus, tests in test points are instrumented 
accordingly for failure detection. TEAMS ${ }^{\circledR}$ can also include information regarding setup operations that need to be performed and resources that are needed, to perform a certain test and can optimize the diagnostic strategy subject to these constraints.

Effects Points and Effects are similar to test points and tests in that they represent locations where failure manifestations are observable. Unlike tests, these failure manifestation points are not instrumented and are typically directly observable. Effects can be categorized as local, isolation/component level or system-wide and have criticality levels associated with them that can differ based on the operational mode of the system. Effects nodes are optional and need to be included only for Probabilistic Risk Assessment (PRA) analyses such as FMECAs and Fault Trees.

The AND node captures redundancy information. For example, if both components A and B has to fail before their failure effects can propagate and affect component $\mathrm{C}, \mathrm{A}$ and $B$ will be connected to $C$ via an AND node. AND nodes allow us to model fault-tolerant systems for diagnosis, reliability and PRA. AND nodes can represent multiple levels of redundancies and can indicate the redundancy as m-out-of-n inputs, where $\mathrm{m} \leq \mathrm{n}$. AND nodes can also be utilized as interim effects that can occur when the redundancy is breached by upstream failures, and removes the need to add an Effect node explicitly at the output of the AND node.

The SWITCH node captures conditional connections or changes in interconnections due to operational mode changes of the system. Switches let us model dynamic and reactive systems whose functional failure propagation may change depending on its operational mode, e.g., an aircraft taxiing, full power take-off and cruising are all different operational modes, and failure modes, links, failure manifestations and their criticalities are likely to be different for these different operational modes of the system.

The aforementioned nodes are interconnected using links, forming a hierarchical graph. Failure propagation algorithms convert this graph to a single global fault dictionary (or Dmatrix), for a given operational mode and state of the system. This D-matrix contains the basic information needed to interpret test results and diagnose failures (onboard monitoring) and generate optimized test sequence that minimizes the troubleshooting time (field maintenance).

Finally, Functions are attributes or tokens that are explicitly added to model and assigned to failure modes, tests and effects. Though not a required construct for a TEAMS ${ }^{\circledR}$ model, Functions allow the representation of functional failures of the system and hence they can be assigned to appropriate failure modes and to the tests and effects which are manifestations of those "failed" functions. Functions can be marked as Diagnostic and/or Effect type as well as Local, where the Local attribute restricts the propagation or effect of the failed function to a specific module hierarchy relative to its association with its failure mode.

TEAMS ${ }^{\circledR}$ toolset for fault management design and operations QSI's TEAMS ${ }^{\circledR}$ Tool Set is widely used in NASA for Systems Engineering, functional failure analysis (Kurtoglu, Johnson, Barszcz, Johnson \& Robinson, 2008), real-time diagnosis during prelaunch checkout (Schwabacher, Martin, Waterman, Oostdyk, Ossenfort \& Matthews, 2010)(Ferrell, Lewis, Perotti, Oostdyk, Spirkovska, Hall \& Brown, 2010) as well as commercial enterprises for Guided Troubleshooting. QSI's TEAMS $^{\circledR}$ Tool Set (Figure 1 - www.teamqsi.com) consists of the following software applications: TEAMSDesigner $^{\circledR}$, TEAMS-RT ${ }^{\circledR}$, TEAMATE $^{\circledR}$ and TEAMS-RDS ${ }^{\circledR}$. The TEAMS ${ }^{\circledR}$ model of a system is a dependency model that captures relationships between failure modes of the system and their observable effects. The model is created in TEAMS-Designer $^{\circledR}$ or imported into TEAMS-Designer ${ }^{\circledR}$ from other data capture environments, and then analyzed and converted into run-time versions for export to the run-time reasoners TEAMATE ${ }^{\circledR}$ and TEAMS-RT ${ }^{\circledR}$. TEAMATE ${ }^{\circledR}$ and TEAMS-RT ${ }^{\circledR}$ are integrated into the TEAMS-RDS ${ }^{\circledR}$ server environment that provides a scalable framework for large number of concurrent health monitoring and guided troubleshooting applications.

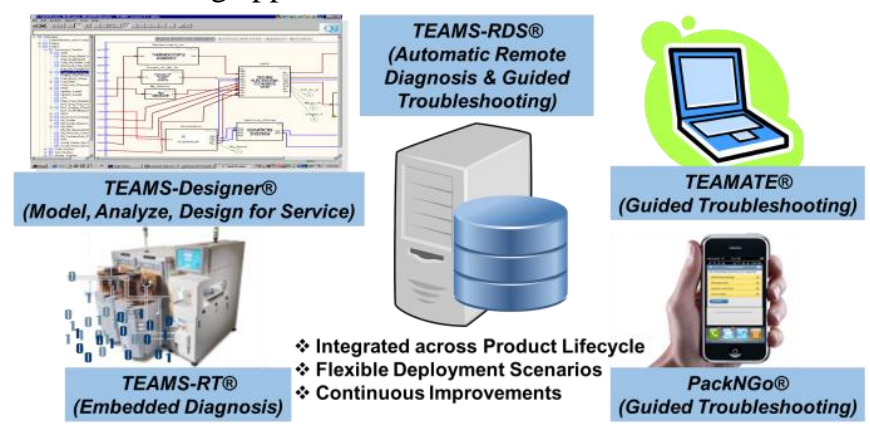

Figure 1: TEAMS $^{\circledR}$ Integrated Toolset

The TEAMS-Designer ${ }^{\circledR}$ application provides a graphical environment for developing dependency models of systems while allowing the specification of several additional practical aspects about the system that are required by the run-time inference engines to provide efficient diagnosis. It does so by allowing the modeler to specify cause-effect dependencies using a hierarchical, multi-layered (multifunction), directed-graph representation of the system (details on QSI's toolset can be found at www.teamqsi.com).

The multi-function modeling methodology (Deb, Pattipati, Raghavan, Shakeri \& Shrestha, 1994) used in $\mathrm{TEAMS}^{\circledR}$ can be conceptualized as a 'colored' dependency graph, where, as in structural models, the structural components are modeled as nodes and the directional links denote dependencies based on structural adjacency. In addition, the system functions are layered onto the nodes as Functions. Thus, if a structural component is responsible for a functionality (denoted by symbol $s$ ), then $s$ is declared a 
Function and layered onto the node denoting that component. Since a multi-function model is based on a structural model, it can model hierarchy of subsystems, line replaceable units, shop replaceable units, etc.

The interconnections of tests (which can be on-board BIT and Error codes, automated, visual indictors, manual troubleshooting), and faults (at different levels of system hierarchy) gleaned from the graphical model, coupled with repair-procedures, enable the modeler to conduct Analysis \& Optimization on the System, generate FMECA Reports, carry out a Fault Tree Analysis and generate a diagnostic strategy for the System. After the model specification is complete, a reachability analysis can be performed in TEAMS-Designer ${ }^{\circledR}$ to internally generate the dependency matrix model of the system subject to specified analysis constraints. TEAMS ${ }^{\circledR}$ Toolset makes use of a common diagnostic model that is used from design through all phases of system operation. Use of the same model across all system disciplines and maintenance phases ensures efficient and seamless transfer of diagnostic and prognostic knowledge, avoid duplication of effort, and prevent any expectation gap between analysis and implementation.

The Run-time Modules: TEAMS-RT ${ }^{\circledR}$ and TEAMATE ${ }^{\circledR}$ and the TEAMS-RDS ${ }^{\circledR}$ framework: Once the dependencymatrix model is available, diagnosis becomes the process of using the dependency relationships and the observed failures or anomalies to infer their possible causes. The functional requirements of the reasoning engine that performs the diagnostic inference depend on the manner in which the observations about the system's state become available. The TEAMS-RT ${ }^{\circledR}$ inference engine processes failure events (error codes, built-in-test failures etc.), as they become available. It uses the data to infer the status of the root causes (the identification of one or more component faults). Thus, TEAMS-RT ${ }^{\circledR}$ is appropriate for processing onboard data that is either received in real time or downloaded postmission/operation.

The TEAMATE ${ }^{\circledR}$ diagnostic reasoner performs inference of component health status as well as computes an optimal sequence of (active) tests that needs to be performed for fault isolation, given the current inferred health status, the allowable set of tests, and any precedence constraints on the tests. Thus, TEAMATE ${ }^{\circledR}$ is appropriate for ground-based deployment where troubleshooting is performed interactively. It is a reasoner in a true sense in that it provides decision support by making recommendations without requiring that the decision maker actually follow its recommendations: it allows the user to decline to perform a recommended action and request an alternative (next best) recommendation. PackNGo ${ }^{\circledR}$ is a mobility agent that extends the Guided Troubleshooting functionality to smartphones (iPhones and Android) and tablets (iPads and Android) over a wireless or Wi-Fi connection.
TEAMS-RDS $^{\circledR}$ (Remote Diagnosis Server) is an Enterprise level application that performs the functions of request broker and load balancing and can support thousands of simultaneous diagnostic sessions from several remote locations, including handheld devices. The TEAMATE ${ }^{\circledR}$ reasoning engine that is served by the TEAMS-RDS ${ }^{\circledR}$ server is designed to provide Interactive Electronic Technical Manuals (IETM) with the capability to support efficient troubleshooting. The TEAMS-RDS ${ }^{\circledR}$ framework provides a relational database for the management of diagnostic and troubleshooting session data, as well as for storing the system models for various equipment configurations.

\subsection{TEAMS ${ }^{\circledR}$ approach towards diagnostic and prognostic health assessment}

While the generally anticipated outcome from prognostic schemes is the Remaining Useful Life (RUL), organizations responsible for system operations and maintenance can be better served with an estimate of the window of opportunity for Preemptive Maintenance (see Figure 2), which can directly lead to actionable decisions related to operations and maintenance. The ability to determine this window of opportunity for Preemptive Maintenance enables the operations or maintenance organization to align recommended maintenance actions within their existing scheduled maintenance cycles, thereby avoiding any additional downtime.

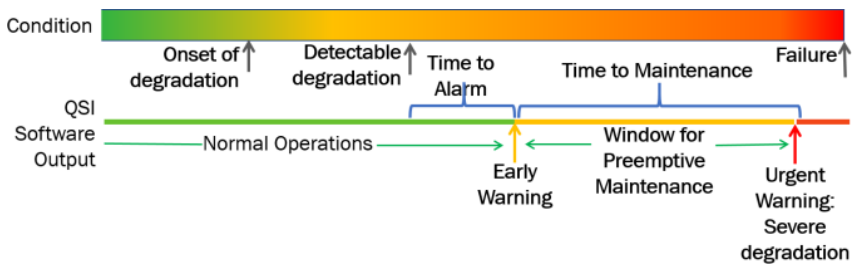

Figure 2: Visual representation of Time-to-Maintenance

QSI's TEAMS ${ }^{\circledR}$ solution generates an Early Warning signal based on a persistent failure of a degradation detection test. The Early Warning signal may be preceded by a Time-toAlarm (TTA), which is an estimate of the time from when the trended deviation was first detected to when the actual failure occurs. Using the inherent TEAMS ${ }^{\circledR}$ cause-effect reasoning capabilities and fusion of inferences from the results of all degradation and failure diagnosis tests, TEAMS $^{\circledR}$ isolates the root cause(s) of the degradation and estimates the Time to Maintenance (TTM) for those root cause(s). TTM is the estimated time to severe degradation or a hard failure from the time of the Early Warning. Absent any mitigating action, and with further system usage, the degradation level and the failure probabilities are continuously evaluated and the TTM is updated accordingly. Once the Window for Preemptive Maintenance elapses, TEAMS ${ }^{\circledR}$ outputs an Urgent Warning, which is an indication that the root causes need immediate attention if an unscheduled downtime event is to be avoided. 
QSI has integrated the approach for TTA and TTM determination in their TEAMS ${ }^{\circledR}$ software suite. The approach uses existing diagnostic capabilities of TEAMS ${ }^{\circledR}$ and newly added tracking and trending algorithms that can detect and track degradation signatures (as observed parameters) in a system. Figure 3 shows a high-level view of TTM determination in TEAMS ${ }^{\circledR}$ of an Electromagnetic Actuation (EMA) System on a certain unmanned helicopter. The view provides a drill-down capability and allows identification of the specific degraded components of the system and their respective TTMs.

\section{QSI nins $\quad$ System liealith Statils}

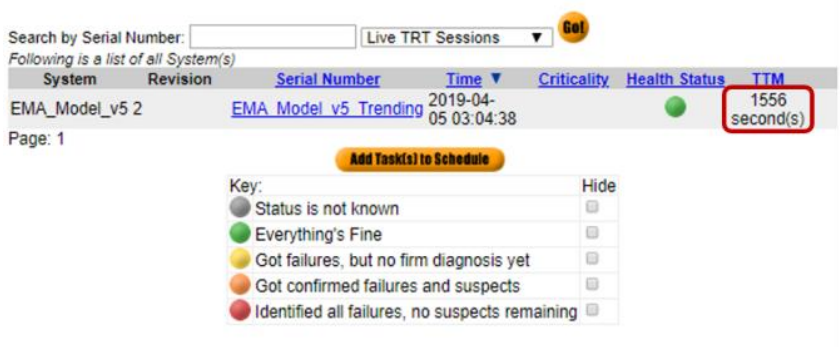

Figure 3: Time-to-Maintenance in $\mathrm{TEAMS}^{\circledR}$ Health Status Dashboard

The key tenets of this approach are as follows:

- Tests can be designed to detect degradation: Observations (or features extracted from observations) from a health monitoring system are typically associated with hard failure(s). Oftentimes, these features can also be associated with degradation detection. Thus, similar test-result evaluation mechanisms can be used for detection of degradations. In such cases the thresholds are usually lower than those for hard failures, in order to detect the onset of degradation early enough.

- Domain and application neutral algorithms can be used to track the "degradation to fault progression": These algorithms follow the trend(s) of the observations (or extracted features) and forecast them over time. The degradation detection tests observe these forecasted trends towards hard failure, and compute the corresponding TTA. These algorithms can be purely self-trained from the observations and do not require any tuning with domain or application-specific knowledge.

- Identify components that will reach significant degradation level within a given time interval: The many-to-many mapping between degradation sources (components) and the degradation detection tests, which is akin to the model-driven paradigm for diagnosis, can be solved using standard diagnostic models and algorithms. The TTA estimates from one or more degradation detection test associated with a degradation source can be fused to estimate its TTM.

The TEAMS ${ }^{\circledR}$ framework is extensible in that third-party prognostics schemes can be integrated within these degradation tests via "plug-in" mechanism. Any TTA estimates from these schemes can also be fused with other degradation tests in the model to provide component TTMs.

\subsection{Application of TEAMS ${ }^{\circledR}$ Modeling for Smart Manufacturing}

The following is an example of successful application of the TEAMS $^{\circledR}$ modeling methodology for Smart Manufacturing Initiative at the Warner Robins Air Logistics Complex (WRALC).

Currently QSI is working with WR-ALC towards deployment of TEAMS ${ }^{\circledR}$ technology for Fault Management of infrastructure-critical complex equipment at the machine shop floor used for depot maintenance of various aircraft parts. The TEAMS ${ }^{\circledR}$ technology is being utilized for identification of critical failure modes of infrastructurecritical complex equipment at the machine shop floor used for depot maintenance through rapid generation and update of Failure Mode Effects and Criticality Analysis (FMECA) reports.

Development of an accurate FMECA, that can be readily updated as the equipment configuration and usage evolve with depot maintenance needs, is a key benefit that can be realized through model based automated FMECA development using TEAMS ${ }^{\circledR}$. An accurate FMECA is also an essential first step towards effective Condition-Based Maintenance (CBM) of that equipment, and TEAMS ${ }^{\circledR}$ software suite provides a natural and consistent progression from FMECA analysis to real-time equipment health monitoring and intelligent, user role-aware, guided troubleshooting, proactive and predictive maintenance of the equipment for both scheduled and unscheduled maintenance events.

Achievement of these objectives identified by the WR-ALC stakeholders will significantly improve the availability and uptime of the infrastructure-critical machines, thereby ensuring that mission-critical aircraft parts being refurbished/renovated or manufactured at WR-ALC facility will be accurately machined and delivered in time whether for scheduled aircraft overhauls or for unscheduled failure events allowing improved return-to-mission for those aircraft. WR-ALC has selected the newly added large, complex and state-of-the-art MAKINO T4 titanium parts machining equipment for model-based FMECA development and Condition-Based Maintenance using TEAMS $^{\circledR}$ technology.

\subsubsection{Model Development}

As part of a key initial step for the equipment model development, QSI acquired the following types of knowledge 
about the system that are needed for the core model constructs as defined earlier:

1) Theory of Operations of the MAKINO T4

2) Overview of the hierarchical physical and functional architecture of the MAKINO T4

3) Various subsystems and their constituent components, their functions and how they fail

4) Associated error codes (Alarms/Warnings etc.) that manifest for those component failures and their failure modes

5) Failure effects especially as they relate to operations that result from those component failures and their failure modes

6) Existing troubleshooting processes and steps when those error codes are triggered

Acquiring the key knowledge attributes required for the failure-cause effect model development using TEAMS ${ }^{\circledR}$ does not necessarily require a deep understanding of the "physics" of the system. The theory of operations typically is sufficient for the understanding of the failure-space related knowledge required for adequate TEAMS $^{\circledR}$ model development. Furthermore, TEAMS ${ }^{\circledR}$ modeling approach only necessitates the enumeration of the component-level functions associated with its failure modes and typically such basic components are well-understood in terms of their failure modes and functions. Thus, any complex equipment such as the MAKINO T4 when decomposed into its constituent components with their own failure modes and functions can be easily determined from a high-level understanding of the theory of operations of the equipment. The above system knowledge requirements for TEAMS ${ }^{\circledR}$ model development, thereby, makes it significantly more straightforward, intuitive and rapid compared with other techniques which impose a significant knowledge barrier for model development.

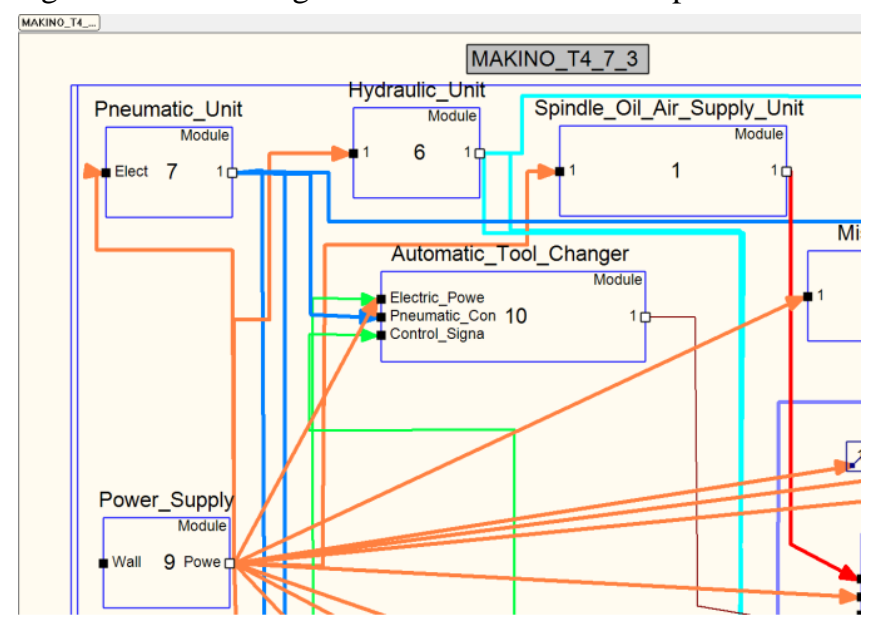

Figure 4: Top-level view (partial) of the MAKINO T4 model in TEAMS Designer
Relying primarily on the MAKINO T4 Theory of Operations manual, a few schematics that shows the component and subsystems layouts ("structural connectivity") and QSI's engineering knowledge of basic components such as power supplies, electronics, pneumatics, hydraulics, pumps, motors, gears, bearings etc., QSI constructed a comprehensive model of the entire equipment and its subsystems, corresponding components and failure modes, as well as various automatic tests/alarms and effects.

The failure-cause effect multi-function model (Figure 4) of the equipment captures the following information necessary for the automation of Failure Modes, Effects, and Criticality Analysis:

- The component and test hierarchy, and hence the Indenture Level for the FMECA analysis

- The failure modes of each of the components

- The reliability (MTTF or failure rate) of each component

- The failure criticality of each component

- Elementary functions performed by each component via the functions attached to each of its failure modes

- Effects of the failures of components, in terms of functional failures and their associations with the alarms/effects/tests that detect them

- Redundancies in the system modeled via AND nodes which allow M-out-of-N switching logic, used to compute whether a failure effect impacts system performance or is masked by redundancy

- The connectivity of components that helps establish cause-effect relationships - this is based on the theory of operations and schematics

- Operational modes of the equipment which is equivalent to the switch and system modes in TEAMS $^{\circledR}$ multifunction models

QSI followed a three-step procedure for TEAMS ${ }^{\circledR}$ modeling that should be adequate for similar modeling needs:

1. Enter the structural model, schematic model or a conceptual block diagram. While in TEAMS ${ }^{\circledR}$, the structural model can be automatically generated from structural models or netlists (e.g., VHDL, EDIF), in this case, the absence of such information led to us directly entering the top-level as well as the hierarchical structure of the system and their physical and functional connectivity using the TEAMS $^{\circledR}$ graphical user interface.

2. Add high-level functions and assign them to the component failure-modes, effects and tests. The set of functions can be identified from the functional specification or the knowledge of the component and its function in the equipment. The failure modes get assigned those functions that are directly impacted by that failure mode. Likewise, the Effects 
and Tests also get assigned those functions whose failures can lead to that Effect occurring or Test failing.

3. Update models with additional information. For example,

- Identify and model the redundant components using AND nodes.

- Identify and model the modes of operations using SWITCHes.

- Provide additional test information, such as setup operations, resource requirements, confidence, diagnostic run levels, etc.

- Identify and indicate functions that are local or transformed, and group functions for clarity.

4. Validate the model. This is a critical step, since the analysis results can only be as good as the models. In TEAMS ${ }^{\circledR}$, the user can interactively seed faults and identify affected tests/effects and vice-versa. Validating a key set of these Dependency Reports that capture the direct failure-cause and effect relationship for most purposes is sufficient to validate the overall model of the system. In addition, peer review and actual integration with run-time tools (i.e., TEAMATE and TEAMS-RT) also provide invaluable feedback on the accuracy of the model

\subsubsection{Analysis Results}

The current version of the MAKINO T4 model has over 300 components with nearly 600 different failure modes. The equipment model is up to 8 hierarchy levels deep, which reflects the complexity of some of the key subsystems of the equipment such as the subsystem that contains the entire Spindle Head and the drives for various movement axes.

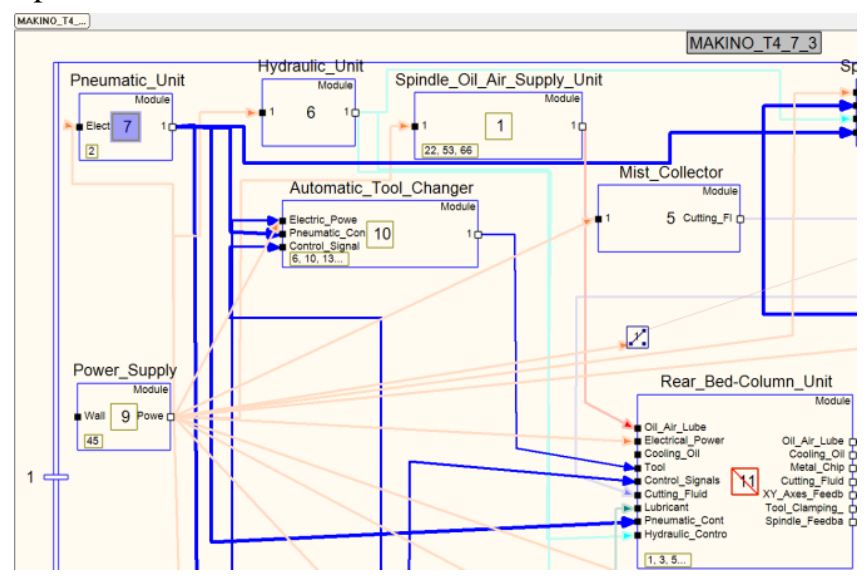

Figure 5: Cross subsystem Pneumatic Unit failure propagation (partial view) in MAKINO T4 as shown in TEAMS-Designer ${ }^{\circledR}$
Figure 5 shows an example of the cross-subsystem failure propagation for the equipment. A solenoid valve leak failure seeded in the Pneumatic Unit leads to the problems with Feed Axes lubrication of the equipment which in turn can result in inaccurate metal cutting by the spindle. The visual representation outlines the various paths of the failure propagation and how it can eventually lead to various toplevel effects including leading to Inaccurate_Cutting.

\begin{tabular}{|c|c|c|c|c|c|}
\hline \multirow[t]{2}{*}{ FUNCTION } & \multirow{2}{*}{$\begin{array}{c}\text { FAILURE } \\
\text { MODES AND } \\
\text { CAUSES }\end{array}$} & \multirow{2}{*}{$\begin{array}{l}\text { MISSION PHASE/ } \\
\text { OPERATIONAL } \\
\text { MODE }\end{array}$} & \multicolumn{3}{|c|}{$\begin{array}{l}\text { FAILURE EFFECTS } \\
\end{array}$} \\
\hline & & & \begin{tabular}{|c|} 
LOCAL \\
EFFECTS
\end{tabular} & $\begin{array}{c}\text { NEXT } \\
\text { HIGHER } \\
\text { LEVEL }\end{array}$ & \begin{tabular}{|c|c|} 
END \\
EFFECTS
\end{tabular} \\
\hline $\begin{array}{l}\text { Slideway_Lubricat } \\
\text { ion }\end{array}$ & \begin{tabular}{|l}
$\begin{array}{l}\text { Z-Axis_Lubrica } \\
\text { nt_Not_Flowin } \\
\mathrm{g}\end{array}$ \\
\end{tabular} & All_Phases & & & \\
\hline $\begin{array}{l}\text { Z-Axis_Power_Su } \\
\text { pply, Movement } \\
\text { Z-Axis, Z-Axis_N } \\
\text { o_Movement }\end{array}$ & $\begin{array}{l}\text { Z-Axis_Servo_ } \\
\text { Amplifier_nnco } \\
\text { rect_offset }\end{array}$ & All_phases & & & \\
\hline $\begin{array}{l}\text { Z-Axis,Power_Su } \\
\text { pply, Movement } \\
\text { Z-Axis, Z-Axis_N } \\
\text { o_Movement }\end{array}$ & \begin{tabular}{|l} 
Z-Axis_Servo \\
Amplifier_Leak \\
age_Current
\end{tabular} & All_Phases & & & \\
\hline $\begin{array}{l}\text { WSS1_Positioning } \\
\text { 'WSS } 2 \text { _Positionin } \\
\text { g, Palle__Change_ } \\
\text { Operation }\end{array}$ & $\begin{array}{l}\text { Pallet_Placeme } \\
\text { n__operator_Er } \\
\text { ror }\end{array}$ & All_phases & 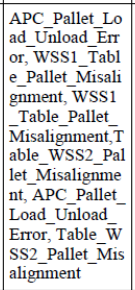 & & $\begin{array}{l}\text { Cannot_Perfor } \\
\text { m_Pallet_Trans } \\
\text { port, Pallet_Cha } \\
\text { nge_Not_Comp } \\
\text { leted_In_Specif } \\
\text { ied_Time, APC } \\
\text { irm_Aotation } \\
\text {-Not_Satisfied, } \\
\text { ANPC_Arm_Do } \\
\text { wn_Not_Satisfi } \\
\text { ed }\end{array}$ \\
\hline
\end{tabular}

Figure 6: Snippet from the automatically generated FMECA report using TEAMS-Designer ${ }^{\circledR}$

Figure 6 shows a snippet from the FMECA document automatically generated by TEAMS-Designer ${ }^{\circledR}$. The document is in the MIL-STD 1629-A format but can be easily adapted to other formats given that the relevant model constructs likely can satisfy most prevalent FMECA formats such as SAE J1739. The display and formatting mechanism in TEAMS-Designer ${ }^{\circledR}$ involves creation of the document in $\mathrm{XML}$ and the application of a stylesheet to generate the PDF version.

Another way to represent the above FMECA-type information is through the automatic generation of the Dependency Report from TEAMS ${ }^{\circledR}$ (Figure 7). Dependency Reports capture the correspondence between an Effect or Test and its corresponding upstream failure mode causes. TEAMS-Designer ${ }^{\circledR}$ computes how failure modes propagate through the system and manifest themselves at various Effect points and Test points, where the occurrences of component failure modes result in those Effects and Tests showing up as detection points for those failure modes. As part of initial validation of the model, we generated Dependency Reports for multiple key spindle related effects and have utilized subject matter expertise to validate those dependency relations. Validation of these dependency relations for a sufficiently large subset of such effects and tests with significant coverage is also how NASA has typically validated its TEAMS $^{\circledR}$ models for spacecraft systems and rockets. 
TEST DEPENDENCY REPORT for MAKINO_14_7_3

Test Name: Y-Axis_Limit_Switch_Position[2]־Rear_Bed-Column_Unit [11]

\begin{tabular}{|c|c|c|}
\hline & Name & MTTF \\
\hline 1 & Y-Axis_Brake [7] - Y-Axis $[3] \leftarrow$ Rear_Bed-Column_Unit $[11]$ & le+06 \\
\hline 2 & Y-Axis_DEC_Incorrect_Reading [1] $\leftarrow$ Limit_Switch_Y-Axis_Dec [8] -Rear_Bed-Column_Unit $[11]$ & le+06 \\
\hline 3 & Y-Axis_DEC_Malfunctioning_Switch [2] $\leftarrow$ Limit_Switch_Y-Axis_Dec [8] - Rear_Bed-Column_Unit $[11]$ & le+06 \\
\hline 4 & Y-Axis_OT2_Incorrect_Reading $[1] \leftarrow$ Limit_Switch_Y-Axis_OT2 [9]־Rear_Bed-Column_Unit $[11]$ & 1e+06 \\
\hline 5 & Y-Axis_OT2_Malfunctioning_Switch [2]—Limit_Switch_Y-Axis_OT2 [9]—Rear_Bed-Column_Unit [11] & $1 \mathrm{e}+06$ \\
\hline 6 & Y-Axis_Nut_Damaged [1] - Y-Axis_Nut $[1] \leftarrow$ Y-Axis $[3] \leftarrow$ Rear_Bed-Column_Unit $[11]$ & 1 e+06 \\
\hline 7 & Y-Axis_Ball_Screw_Damaged [1] - Y-Axis_Ball_Screw [2] - Y-Axis $[3] \leftarrow$ Rear_Bed-Column_Unit [11] & $1 \mathrm{e}+06$ \\
\hline 8 & Y-Axis_Disk_Spring_Damaged [1] - Y-Axis_Disk_Spring [3] $[$ Y-Axis [3] Rear_Bed-Column_Unit [11] & $1 \mathrm{e}+06$ \\
\hline 9 & Y-Axis_Bearings_Damaged $[1] \leftarrow$ Y-Axis_Bearings $[4]-Y$-Axis $[3] \leftarrow$ Rear_Bed-Column_Unit $[11]$ & $1 \mathrm{e}+06$ \\
\hline 10 & Y-Axis_Coupling $[1] \leftarrow$ Y-Axis_Motor_Coupling [5] - Y-Axis [3]-Rear_Bed-Column_Unit $[11]$ & 250000 \\
\hline 11 & Y-Axis_Clamp_Ring [2] & le+06 \\
\hline
\end{tabular}

Figure 7: A segment of the Dependency Report showing Failure Causes for the "Spindle Did Not Start" Effect

Figure 7 shows an example of a Dependency Report for the Effect "Spindle Did Not Start" that lists all the different Failure Modes, each of whose occurrence can lead to this Effect. It appears that this Effect can be caused by a large number of Failure Modes across the entire MAKINO T4 equipment components. Failure Modes from different subsystems such as the Automatic Pallet Changer (APC), lubrication subsystems such as the Spindle Oil Air Supply Unit, Tool Clamping Mechanisms, Power Supply can all cause this Effect to manifest itself.

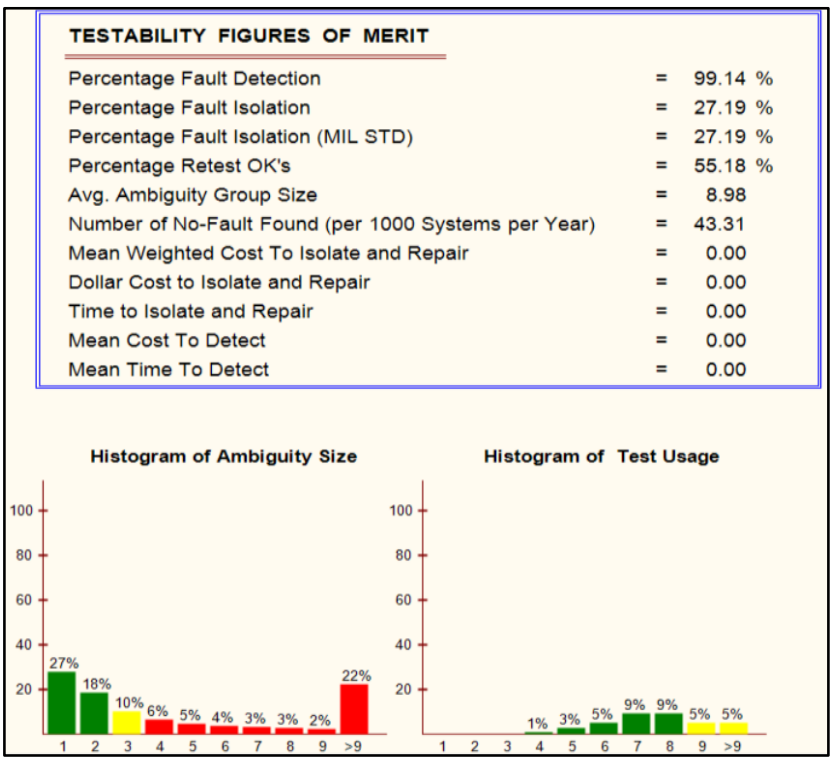

Figure 8: Testability Figures of Merit (TFOMS) report (a partial view) for the MAKINO T4 model

Testability Analysis (Figure 8) performed on the model using all diagnostic tests with isolation set to the component level, shows a percentage fault detection over $99 \%$ but a low
Percentage Fault Isolation at about $27 \%$, i.e., only $27 \%$ of the components can be uniquely isolated as root causes for failure events. The Testability Analysis figure also shows the current distribution of the ambiguity group sizes, i.e., the percentage of groups of components $(1,2,3$ etc.) cannot be further isolated. For example, the histogram shows that $27 \%$ of the components are fully isolateable, $18 \%$ of them can be isolated to a size of two components, a group size of 3 happens for $10 \%$ of the components, etc. As the focus of the model development effort changes towards improving Guided Troubleshooting and more manual tests are added the Fault Isolation metrics are expected to improve substantially.

\section{Conclusions}

Significant life cycle cost savings can be achieved via an integrated model-based system engineering approach towards Fault Management that addresses all of the equipment life-cycle spanning Design for Testability/Serviceability, FMECA generation, health monitoring, guided troubleshooting and prognostics. This requires a common knowledge representation (i.e., TEAMS ${ }^{\circledR}$ multi-function cause-effect dependency models in TEAMSDesigner $^{\circledR}$ ) that can be used at every stage of the system lifecycle, and a set of diagnostic reasoning engines (i.e., TEAMS $^{\circledR}$ toolset) that implements those solutions for realtime health assessment for onboard diagnostics deployment, guided troubleshooting and condition-based maintenance. This paper is a brief overview of our efforts to achieve such a comprehensive solution.

\section{REFERENCES}

Kurtoglu T., Johnson S. B., Barszcz E., Johnson J. R. \& Robinson P. I. (2008). Integrating System Health Management into the Early Design of Aerospace Systems Using Functional Fault Analysis. Prognostics and Health Management Conference, Denver, CO.

Schwabacher M., Martin R., Waterman R., Oostdyk R., Ossenfort J. \& Matthews B. (2010). Ares I-X Ground Diagnostic Prototype. AIAA Infotech Conference, Atlanta, GA.

Ferrell R., Lewis M., Perotti J., Oostdyk R., Spirkovska L., Hall D. \& Brown B. (2010). Usage of Fault Detection Isolation \& Recovery (FDIR) in Constellation (CxP) Launch Operations. AIAA SpaceOps Conference, Huntsville, AL.

Deb S., Pattipati K. R., Raghavan V., Shakeri M., \& Shrestha R. (1994). Multi-Signal flow graphs: a novel approach for system testability analysis and fault diagnosis. Proc. IEEE AUTOTESTCON, Anaheim, CA, pp. 361-373. 


\section{BIOGRAPHIES}

Sudipto Ghoshal, Ph.D., VP of Engineering at Qualtech Systems, Inc. (QSI), received his B. Tech degree in Electrical Engineering from the Indian Institute of Technology, Kharagpur, India in 1989, the M.S. and Ph.D. degrees in Biomedical Engineering from the University of Connecticut, Storrs in 1991 and 1997, respectively and an MBA from Indiana University, Kelley School of Business, Bloomington in 2009. His research at QSI primarily involves developing and implementing algorithms for highly scalable, compact diagnostic reasoning engines and architecting the test implementation modules for the TEAMS-RDS ${ }^{\circledR}$ software framework. He is a committee member of IEEE SCC20 Diagnostics and Maintenance subcommittee since 1991 and has played an active role in developing standards for system diagnosis. He, along with several colleagues at QSI, holds a patent for inventions related to a distributed architecture for system diagnosis. He has published numerous journal and conference papers and has received several best paper awards in technical conferences. He was a recipient of the 2002 \& 2008 NASA Space Act Award for "A Comprehensive Toolset for Model-based Health Monitoring and Diagnosis."

Somnath Deb, SMIEEE '98, President, CTO at Qualtech Systems, Inc. (QSI), received the M.S. and Ph.D. degrees in Control and Communication Systems from the University of Connecticut (UCONN) in 1990 and 1994, respectively. In 2013, he was inducted into the UConn Academy of Distinguished Engineers for outstanding contributions to the School of Engineering and to the engineering profession. His research interests include Integrated Diagnostics and Vehicle Health Management Architectures and Solutions, embodied in QSI's TEAMS ${ }^{\circledR}$ tool suite for design for service, real-time embedded diagnostics, tele-diagnosis, and guided troubleshooting solutions. He has published over 50 journal and conference papers. He received the Best Technical Paper Awards at the 1990, 1994, 2001 AUTOTEST Conferences and the NASA Space Act Awards in 2002 and 2008 for his work on tools for model-based testability analysis, guided troubleshooting, and remote health monitoring.

Deepak Haste, Director of Engineering at Qualtech Systems Inc., received his Bachelor's Degree in Electrical Engineering from the Indian Institute of Technology, Bombay, India in 1996 in Electrical Engineering and M.S degree in Electrical and Computer Engineering from Clemson University, South Carolina in 1998. He played a prominent role in productizing QSI's TEAMS ${ }^{\circledR}$ Toolset, and functioned as the lead in several customer-driven TEAMS ${ }^{\circledR}$ Toolset enhancements. His key efforts include adding Prognostic capabilities to the TEAMS $^{\circledR}$ Suite as well as combining Machine Learning techniques with Model-driven approaches. He has wide-spread experience in integration of QSI's Tools with third-party software, as well as embedding and interfacing TEAMS $^{\circledR}$ diagnostic software within various onboard platforms. He has successfully lead several key research projects with NASA and DoD that resulted in value-add enhancements to the TEAMS ${ }^{\circledR}$ Toolset.

Andy Hess is a 1969 graduate of the University of Virginia (BS Aerospace Engineering) and the U. S. Navy Test Pilot School. He has completed many Navy and DOD sponsored professional and acquisition management courses. He is world renowned for his work in fixed and rotary wing health monitoring and is recognized as the father of Naval Aviation propulsion diagnostics. Working for the Naval Air System Command and beginning with the A-7E Engine Monitoring System program of the early 70 's, he has been the leading advocate for health monitoring in the Naval Aviation. He has been actively involved in every NAVAIR aircraft program since the F-8, leading to the evolution and development of not just engine monitoring; but also aircraft structural life usage, avionics systems, mechanical systems, and comprehensive health monitoring and management capabilities for most all other aircraft subsystems and advance maintenance concepts like Condition Based Maintenance $(\mathrm{CBM}+)$. For the last 10 years of his government career, he worked leading and managing the vision, the development, and integration of the Prognostic and Health Management (PHM) system the AL support concept for the Joint Strike Fighter F-35 program. His current consulting services and interests are now leading him and his clients to exploring the application of PHM capabilities, predictive analytics, $\mathrm{CBM}+$, and asset management related support concepts to many diverse industry sectors such as: aerospace, industrial gas and steam turbines, ships and fast patrol boats, unmanned vehicles, wind energy, nuclear energy, ground vehicles, mining, and gas and oil. Serving on the Board of Directors, he helped establish and grow the new and very successful PHM Society professional organization and is the current president of the society. He was named an Asset Management Fellow with the International Society of Engineering Asset Management, is a NAVAIR Senior Engineering Fellow, and is a member of the SAE E-32 committee on Propulsions System Health Monitoring, the SAE HM-1 committee on Integrated Vehicle Health Management Systems and is a prognostic lead for the ISO committee on condition monitoring. He has been long and very active as the organizing chair for the Advanced Diagnostic and PHM Track at the annual IEEE Aerospace Conference. He has published a large number significant peer group reviewed technical papers and has co-authored a widely used text book on prognosis of engineering systems. In his current consulting role he has provided extensive support to a sundry of OEM's, government and research organizations, academia, and small businesses. Recently, he has been very engaged with the University of Cincinnati IMS Center in regard with growing and implementing PHM capabilities to Smart Manufacturing and Industry AI applications. Most recently, he has been professionally recognized with a lifetime achievement award for PHM. 
Feraidoon Zahiri, Engineer at the US Air Force, received his Master of Science degree in Engineering Mechanics from Penn State University. He has more than 20 years of experience in technology research, development, and transition in numerous scientific and engineering disciplines. $\mathrm{He}$ has overseen large-scale decision-support projects incorporating analytics, simulation, and visualization for resource allocation, system troubleshooting and repair, and data-driven predictive maintenance. He was selected as a winner of the Small Business Technology Council's (SBTC) 2017 "Champion of Small Business Technology Commercialization" Award, for his role in filling a critical technology gap in the Air Force Sustainment community. He has played a critical role in providing opportunities for small business to promote the technology across all DoD services, boosting their technology transition even further. He has been instrumental in testing and transitioning new technologies developed through the CTMA (Commercial Technologies for Maintenance Activities) program for the past several years including a new advanced ATE (Automated Test Equipment) for testing electrical subsystems and more recently, the application of a new Voice-enabled Inspection System, VIMS (winner of the 2016 CTMA Partners Technology Challenge). He has been a strong government advocate of the CTMA program.

Gregory Sutton, Engineering Director at 402d Commodities Maintenance Group, Warner-Robins Air Logistics Complex, received his Bachelor of Mechanical Engineering at Georgia Institute of Technology in 1993 and Masters of Logistics Administration at Georgia College \& State University in 2009. He began his civilian service in 2002 as support equipment engineer before leading Technology Insertion across Robins AFB then helping create the first, new Airworthiness certification for the CV-22 in 2010. He currently oversees the process, equipment, and facilities engineering for a large depot maintenance industrial operation with an annual labor and material budget of $\$ 147 \mathrm{M}$ across 32 facilities. The Engineering Squadron consists of 65 engineers, technicians, $\mathrm{He}$ was previously the Weapons Engineering Section Chief, Armament Sustainment Division. He led 13 civilian, military, and contract engineers to sustain and modernize all USAF medium caliber $(20 \mathrm{~mm}$ and $30 \mathrm{~mm})$ guns, bomb racks, small arms, and Special Operations weapon $(25 \mathrm{~mm}, 40 \mathrm{~mm}$, and $105 \mathrm{~mm})$ supporting B-1, B-2, B52, A-10, F-15, F-16, F-22, CV-22, and AC-130. He is the recipient of several awards including the USAF Modeling \& Simulation Award (2015), Robins AFB Director's Choice Science, Engineering, \& Technical Management (2015), AF LifeCycle Management Center (AFLCMC) Technical Leadership (2014), and Robins AFB Engineering \& Technical Management Mid-Career Civilian (2008) awards. 